\title{
Rigorous approach to bore-sight self-calibration in airborne laser scanning
}

\author{
Jan Skaloud ${ }^{\mathrm{a}, *}$, Derek Lichti ${ }^{\mathrm{b}}$ \\ ${ }^{\text {a }}$ TOPO Lab, École Polytechnique Fédérale de Lausanne, Station 18, 1015 Lausanne, Switzerland \\ ${ }^{\mathrm{b}}$ Department of Spatial Sciences, Curtin University of Technology, GPO Box U1987, Perth, Australia
}

Received 10 January 2006; received in revised form 20 July 2006; accepted 20 July 2006

Available online 7 September 2006

\begin{abstract}
We present a rigorous method for estimating some of the calibration parameters in airborne laser scanning (ALS), namely the three bore-sight angles and the range-finder offset. The technique is based on expressing the system calibration parameters within the directgeoreferencing equation separately for each target point, and conditioning a group of points to lie on a common surface of a known form such as a plane. However, the assumed a priori information about $q$ chosen planar features is only their form not the spatial orientation or position. Thus, the $4 \cdot q$ plane parameters are estimated together with the calibration parameters in a combined adjustment model that makes use of GPS/INS-derived position and orientation as well as LiDAR range and encoder angle as observations. To make the approach practical when working with voluminous ALS and GPS/INS data, the contribution of each laser point to the normal equations is formed sequentially. The discussions focus on practical examples with data from a continuouslyrotating scanner that reveal the conditions under which almost complete de-correlation between the estimated parameters occurs. In such a case, all bore-sight angles are determined with accuracy that is several times superior to the system noise level. Given sufficiently strong geometry, the presented method is shown to be not only accurate but also very robust in terms of convergence. When appropriate, the method is applicable for calibration of additional systematic effects such as laser-beam encoder offsets or scale factor with minimal modification to the functional model.

(C) 2006 International Society for Photogrammetry and Remote Sensing, Inc. (ISPRS). Published by Elsevier B.V. All rights reserved.
\end{abstract}

Keywords: LIDAR; Calibration; Georeferencing; GPS/INS; Laser scanning; Accuracy

\section{Introduction}

Airborne Laser Scanning (ALS) is a very effective and accurate method for establishing detailed terrain models from airborne platforms. In some applications the requirements on point density and DTM accuracy

\footnotetext{
* Corresponding author. Tel.: +41 21693 2753; fax: +41 216935740.

E-mail addresses: jan.skaloud@epfl.ch (J. Skaloud), d.lichti@curtin.edu.au (D. Lichti).
}

can be as high as several points per $\mathrm{m}^{2}$ and $0.1 \mathrm{~m}$, respectively. The factors affecting laser-target position accuracy are numerous (Schenk, 2001). Apart from the target reflectivity properties and laser-beam incidence angle, the main limiting factors are the accuracy of the platform position and orientation derived from the carrier-phase differential GPS/INS data and uncompensated effects in system calibration. The calibration can be divided into that of calibration of individual sensors such as the laser range-finder and that concerning spatial

0924-2716/\$ - see front matter (C) 2006 International Society for Photogrammetry and Remote Sensing, Inc. (ISPRS). Published by Elsevier B.V. All rights reserved.

doi:10.1016/j.isprsjprs.2006.07.003 
(lever-arm) or orientation (bore-sight) offsets between the sensors due to a particular assembly. In most system installations, the lever-arms between LiDAR-IMU-GPS sensors can be determined separately by independent means, although this represents certain difficulties related to the realization of the IMU body frame. On the other hand, the determination of the bore-sight angles is only possible in-flight once the GPS/INS-derived orientation becomes sufficiently accurate.

Though several well-developed approaches to boresight estimation for camera-IMU installations exist (Kruck, 2001; Cramer and Stallmann, 2002; Skaloud and Schaer, 2003), the correct recovery of the ALS-IMU misalignment still remains elusive. The existing calibration procedures, while functional, are recognized as being sub-optimal since they are labor-intensive (i.e., they require manual procedures), non-rigorous and provide no statistical quality assurance measures (Morin and El-Sheimy, 2002). Furthermore, the existing methods often cannot reliably recover all three of the angular mounting parameters. The undetermined parameter(s) propagate into the subsequently captured data, therefore compromising its accuracy and that of any derived information. Thus, much research effort is devoted to their improvement worldwide. The adopted approaches are usually based either on physical boundaries or cross-sections (Schenk, 2001) or DTM/ DSM gradients (Burman, 2000). Also, Morin and ElSheimy (2002) suggest a photogrammetric calibration approach using many signalized targets (tie points). The drawback of this method is the need for interpolation of tie point coordinates in the ALS data that limits the pointing accuracy and, hence, calibration accuracy. The problem is worsened by the angular uncertainty due to the broad laser beamwidth (Lichti, 2004).

On the other hand, the cross-section method seems to be popular in commercial systems (Optech, 2004) and usually provides satisfactory results for the boresight estimate in the roll direction. However, as will be shown later, its use for the recovery of pitch and yaw/ heading direction is less appropriate. The use of the slope gradients in DTM/DSM for bore-sight estimation made its way to a popular software package used for ALS data handling (Soininen and Burman, 2005). The principal weakness of this approach is the strong correlation of the bore-sight angles with unknown terrain shape. Also, the implemented stochastic model of the LiDAR trajectory assumes time-invariant behavior of the GPS/INS errors that is not realistic. Similar weaknesses also affect earlier methods related to strip adjustment where offsets and rotations are modeled in the object domain (Vosselman and Maas,
2001). The more rigorous class of calibration procedures or strip adjustments uses the modeling of systematic errors directly in the measurement domain (Filin, 2003; Filin and Vosselman, 2004). More recently, Friess (2006) reports a rigorous method also based upon block adjustment principles, but only presents results with simulated data. Although developed independently, the approach presented here builds upon the same basic foundation. As will be seen, though, it develops important differences.

Similar to Filin (2003) we start from correct modeling of systematic effects in the direct georeferencing equations and conditioning the object coordinates of group of points to lie on a common plane. It will be shown that good calibration requires the use of many planar features with different spatial orientation and these are easily identified on urban structures where the access is often limited. Therefore, it is useful to recover the plane parameters together with the calibration parameters in a combined adjustment model. This has an important practical impact as no additional surveying campaign apart from the calibration flight itself needs to be organized for the system calibration. Also, the method uses the aircraft trajectory and laser measurements as observables and therefore can benefit from a rigorous approach to the GPS/INS stochastic modeling and error propagation from range observations to object space coordinates.

The organization of the paper is as follows. After giving definitions of system errors and emphasizing their influence on mapping results, the functional model for their recovery is developed and its linearization is presented. The subsequent discussion concerns the choice of the stochastic model and its practical impact on the computational performance. It will be shown that for a special case the normal equations can be formed sequentially, which has an important practical impact when handling voluminous ALS data sets. The initial values for the plane parameters will be estimated directly from the data by a computationally efficient method. The stability of the solution will then be tested for its sensitivity with respect to the choice of the initial parameters and convergence rate using different data sets. The discussion of the numerical examples will address the conditions that affect the accuracy of and correlation between the estimated parameters. As will be demonstrated this is especially critical for the estimate of the range-finder correction. The synthesis of these investigations supported by numerical examples will lead to important claims as to the suitability of the presented approach for its wide use in ALS system calibration. 


\section{Airborne laser scanning}

\subsection{Direct georeferencing of LiDAR measurements}

As already mentioned, the need for system calibration parameters arises from the LiDAR observations and from their transformation by the GPS/INS data. Using an arbitrary Cartesian mapping frame $(m)$, the direct georeferencing of LiDAR measurements at time $t$ (epoch $i$ ) with GPS/INS on a common platform takes the following form:

$$
\begin{aligned}
{\left[\begin{array}{l}
x \\
y \\
z
\end{array}\right]^{m}=} & {\left[\begin{array}{c}
X \\
Y \\
Z
\end{array}\right]^{m} } \\
& +R_{b}^{m}\left[\left(I+\Omega_{b^{*}}^{\mathrm{b}}\right) T_{s}^{\mathrm{b}^{*}}\left(\begin{array}{c}
(\rho+\Delta \rho) \sin (\theta) \\
0 \\
(\rho+\Delta \rho) \cos (\theta)
\end{array}\right)+\left[\begin{array}{c}
a_{x} \\
a_{y} \\
a_{z}
\end{array}\right]^{b}\right]
\end{aligned}
$$

Thereby,

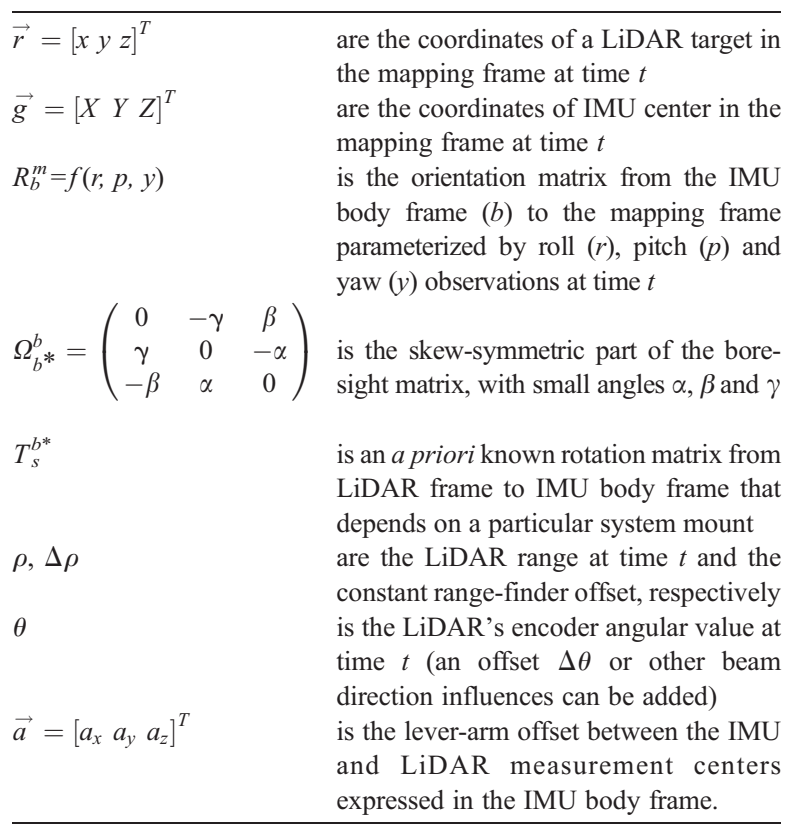

In the above definition, the relative orientation from the LiDAR frame to IMU body frame for a particular system mount ' $M$ ' is decomposed into two subsequent rotations: $M_{s}^{b}=\left(I+\Omega_{b *}^{b}\right) T_{s}^{b *}$ with $T_{s}^{b *}$ representing the approximate axis orientation assumed to be known $a$ priori and $\left(I+\Omega_{b *}^{b}\right)$ being a matrix of small unknown rotations defined as the bore-sight. Although this definition of the bore-sight assumes its maximal uncertainty at the level of few degrees only, this is usually more than practically needed.

\subsection{Definition of calibration parameters}

In Eq. (1), the system calibration parameters due to the system mount are the lever-arm vector $\vec{a}$ and the bore-sight angles $\alpha, \beta$ and $\gamma$. As mentioned earlier, it is assumed that the vector $\vec{a}$ is determined separately by independent means and therefore excluded from the inflight calibration. In contrast, the bore-sight angles are the main subject of the in-flight system calibration as it is difficult to estimate them by other means. The practical influence of the bore-sight on the mapping accuracy is demonstrated by Fig. 1. This figure shows a crosssection of a building recovered from 8 flight lines (two cloverleaf patterns flown at different heights). As can be seen from the profile, the discrepancies due to roll errors (Fig. 1a) are clearly visible on the inclined and horizontal planes. The errors due to pitch (Fig. 1b) are not apparent in horizontal planes; however, they have an opposite effect on inclined planes. Finally, the errors in yaw/ heading (Fig. 1c) cannot be discerned from the profile,
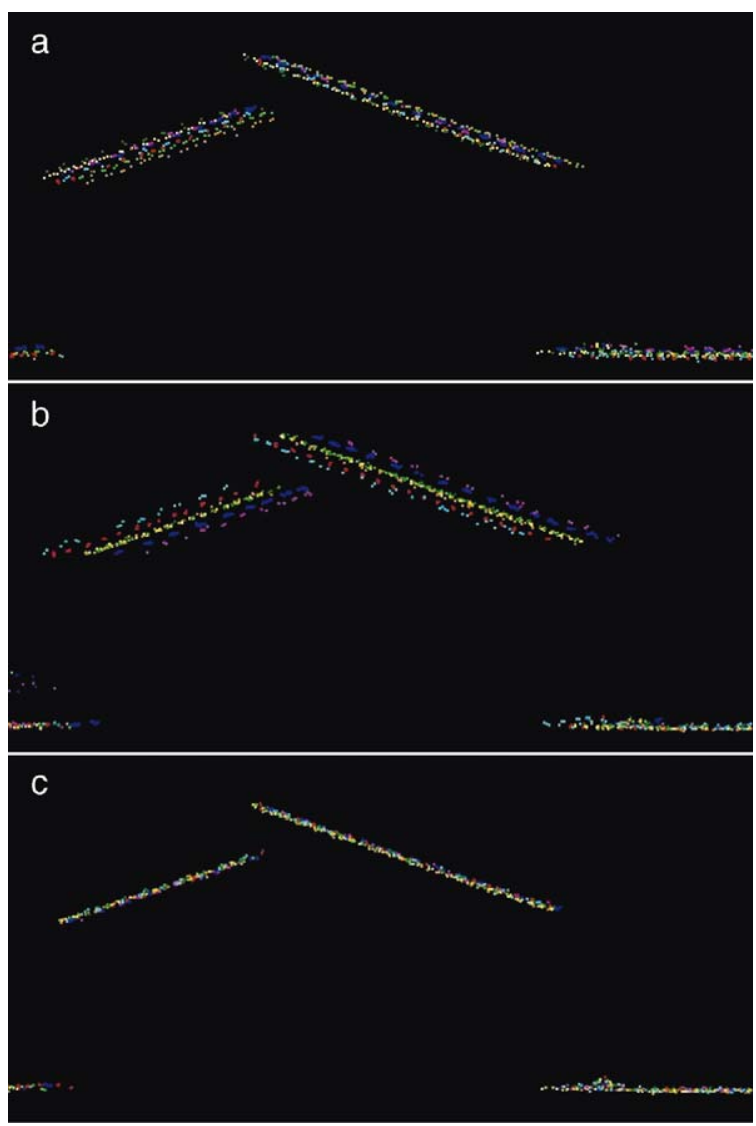

Fig. 1. Influence of bore-sight error on a cross-section plotted separately for the roll (a), pitch (b) and yaw (c) angles from 8 flight lines of different directions and heights. The magnitude of each boresight angle was deliberately set to $0.3^{\circ}$ for each example. 
which is why cross-section analyses are not suitable for recovery of this component of the bore-sight.

The magnitude of systematic errors in LiDAR measurements are influenced by the concept of the scanning principle and the quality of fabrication. Although these errors are best avoided by proper system design (Katzenbeisser, 2003), some residual effects must be expected even after factory calibration. In Eq. (1) we have considered only a range-finder bias; other effects such as the angle-encoder zero offset can be added if appropriate. In addition, a scanning system using a mirror of constant velocity, such as the one tested in this study, does not suffer from non-linear effects due to mirror acceleration; these were therefore omitted.

Additional candidates (at least theoretically) for calibration are the systematic influences in the navigation data. These are sometimes modeled as constant shift and/ or drift when adjusting adjacent strip (Filin and Vosselman, 2004). Although this is a justifiable approach for alleviating remaining artifacts in laser point clouds within a particular survey job, a rigorous approach requires modeling remaining systematic effects in GPS/ INS integration directly within the navigation Kalman Filter. Obviously, taking such an approach would not only complicate the matter but may also degrade the quality of the parameter estimates due to additional correlation. Fortunately, the choice of the calibration area and the flight conditions can usually be optimized in terms of the flight dynamics and satellite constellation to a level that the residual effects in GPS/INS trajectory estimation are lower than $0.05-0.1 \mathrm{~m}$ and $0.005-0.01^{\circ}$ in position and attitude, respectively, when considering the use of dual frequency GPS receivers and a tacticalgrade INS. No additional parameters due to navigation (or timing) errors were necessary in the examples presented herein thanks to optimal flight conditions. These include close separation $(<5 \mathrm{~km}$ horizontally, $<0.4 \mathrm{~km}$ vertically) from the base receiver, optimal satellite constellation, accurate alignment of the inertial system, short flight lines and good estimation of the IMU systematic errors between the flight lines thanks to specifically-flown maneuvers.

\section{Estimation model}

\subsection{Functional model}

The development of the functional model is based on conditioning the georeferenced LiDAR target points to lie on surfaces of known form, particularly planes. The parameters of these planes are estimated together with the calibration parameters. The parameters of a plane $j$ are represented as $\overrightarrow{s_{j}}=\left[\begin{array}{llll}s_{1_{j}} & s_{2_{j}} & s_{3_{j}} & s_{4_{j}}\end{array}\right]^{T}$ where $s_{1}, s_{2}$ and $s_{3}$ are the direction cosines of the plane's normal vector and $s_{4}$ is the negative orthogonal distance between the plane and the coordinate system origin. The observation equation for an object point $i$ expressed by its coordinates $x_{i}, y_{i}, z_{i}$ lying on plane $j$ is given by $s_{1_{j}} x_{i}+s_{2_{j}} y_{i}+s_{3_{j}} z_{i}$ $+s_{4_{j}}=0$ or

$$
\begin{aligned}
\left\langle\vec{s}_{j},\left[\begin{array}{r}
\vec{r}_{i} \\
1
\end{array}\right]\right\rangle & =0=f\left(\vec{\ell}, \vec{x}_{1}, \vec{x}_{2}\right) \\
& =f\left(\vec{\ell}^{\text {obs }}+\hat{\delta}_{1}, \vec{x}_{1}^{0}+\hat{\delta}_{1}, \vec{x}_{2}^{0}+\hat{v}\right) .
\end{aligned}
$$

In the latter equation, the position vector of observed point $i$ on the plane $\overrightarrow{r_{i}}=h\left(\vec{\ell}_{i}, \vec{x}_{1}\right)$ is a function of the GPS/INS trajectory estimates and two LiDAR observa-

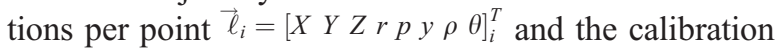
parameters $\overrightarrow{x_{1}}=[\alpha \beta \gamma \Delta \rho]^{T}$, with all symbols defined previously; $\vec{x}_{2}$ is the vector of plane parameters spanning $q$ planes of interest; the superscript " 0 , denotes the parameters initial values and the superscript " $"$ the estimated quantities which are the corrections to the calibration $\left(\delta_{1}\right)$ and plane $\left(\delta_{2}\right)$ parameters and the residual corrections $\hat{v}$ of the 8 observations per point. Using the following definitions

$\vec{u}=\left[\begin{array}{lll}u & v & w\end{array}\right]^{T} \equiv T_{s}^{b^{*}}\left[\begin{array}{c}(\rho+\Delta \rho) \sin \theta \\ 0 \\ (\rho+\Delta \rho) \cos \theta\end{array}\right]$

and

$U \equiv\left(\begin{array}{ccc}0 & w & -v \\ -w & 0 & u \\ v & -u & 0\end{array}\right)$

substitution of the LiDAR georeferencing Eq. (1) into the observation Eq. (2) leads to the desired form of the functional used for the parameter estimation:

$\left\langle\overrightarrow{s_{j}},\left[\left[\begin{array}{c}X_{i} \\ Y_{i} \\ Z_{i}\end{array}\right]+R_{b_{i}}^{m}\left(\left[\begin{array}{c}u_{i} \\ v_{i} \\ w_{i}\end{array}\right]+U_{i}\left[\begin{array}{c}\alpha \\ \beta \\ \gamma\end{array}\right]+\vec{a}\right)\right]\right\rangle=0$

Note that the direction cosines must satisfy the following unit length constraint

$s_{1_{j}}^{2}+s_{2_{j}}^{2}+s_{3_{j}}^{2}-1=0=g\left(\vec{x}_{2}\right)$

For $p$ points and $q$ planes, then the following basic quantities can be formed:

$\begin{array}{ll}\text { \# conditions } & m=p \\ \text { \# unknowns } & u=u_{1}+u_{2}=4+4 q \\ \text { \# observations } & n=8 p \\ \text { \# constraints } & c=q \\ \text { \# degrees of freedom } & r=p-4-3 q\end{array}$


Note that if the range-finder offset, $\Delta \rho$, is not estimated, then the number of unknowns is reduced to $3+4 q$.

\subsection{Linearization of the functional model}

Since the observations and parameters of the pointon-plane observation equation are not separable and each condition includes more than one observation, the combined (or Gauss-Helmert) adjustment model must be used (Förstner and Wrobel, 2004). As there are two sets of unknowns, $\vec{x}_{1}$, and $\vec{x}_{2}$, the linearized system of equations will be partitioned and takes the form:

$A_{1} \hat{\delta}_{1}+A_{2} \hat{\delta}_{2}+\underset{m, n}{B} \hat{v} \hat{v}+\underset{m, 1}{w}=\underset{m, 1}{0}$

where $A_{1}=\partial f / \partial \vec{x}_{1}$ and $A_{2}=\partial f / \partial \vec{x}_{2}$ are the respective design matrices of partial derivatives of the functional (5) taken with respect to the calibration parameters and the plane parameters; $\hat{\delta}_{1}$ and $\hat{\delta}_{2}$ are the respective vectors of corrections to the approximate parameter values; $B=$ $\partial f / \partial \vec{\ell}$ is the design matrix of partial derivatives of the same functional taken with respect to the observations; $\hat{v}$ is the vector of residuals; $w=f\left(\vec{\ell}, \vec{x}^{0}\right)$ is the misclosure vector, i.e. the functional (5) evaluated at current estimates of the parameters and observations.

The constraint (6) on the direction cosines (i.e. their sum of squares must equal unity) is implemented as a weighted parameter constraint, which essentially takes the form of a "classic" observation equation where the observation is zero, i.e. $s_{1_{j}}^{2}+s_{2_{j}}^{2}+s_{3_{j}}^{2}-1=\hat{v}_{c}$. The linearized matrix form of the constraints is given by

$G \delta_{c, u_{2}}+w_{c}=v_{c}$

where $G=\partial g / \partial \vec{x}_{2}$ is the design matrix of partial derivatives of the constraint functional $g$ taken with respect to the plane parameters; $w_{c}=g\left(\vec{x}_{1}^{0}\right)$ is the constraint functional misclosure vector; and $\hat{v}_{c}$ is the constraint residual.

\subsection{Stochastic model}

The influence of considering correlations within the GPS/INS observations for the IMU-camera bore-sight determination is demonstrated by Skaloud and Schaer (2003). There are two types of correlation in the GPS/ INS data. The first is reflected in the correlation between the 6 parameters of the exterior orientation; the other is the time-correlation between the successive estimates. As shown in Skaloud and Schaer (2003), the second is far more significant for optimal bore-sight estimation with respect to the camera and thus most likely also with respect to the laser system. Although similar influences can be expected for the IMU-LiDAR case, the present stochastic model will assume all observational errors (of the eight observations per point and the constraint observations) to be zero-mean and uncorrelated with each other. These assumptions are sub-optimal and will be partly compensated by increasing the time varying covariances of the individual GPS/INS observations. On the other hand, considering purely diagonal covariance matrices allows important simplifications in the model formulation when processing voluminous ALS data. Considering this, the weight matrices, $P$ for the observations and $P_{c}$ for the constraints, are assumed to be diagonal:

$$
\begin{aligned}
P, n & =\left[\begin{array}{cccc}
P_{1} & 0 & \cdots & 0 \\
0 & P_{2} & \cdots & 0 \\
\vdots & \vdots & \ddots & \vdots \\
0 & 0 & \cdots & P_{m}
\end{array}\right] \text { with } \\
P_{i} & =\operatorname{diag}\left(\frac{1}{\sigma_{\mathrm{X}_{i}}^{2}} \frac{1}{\sigma_{\mathrm{Y}_{i}}^{2}} \frac{1}{\sigma_{\mathrm{Z}_{i}}^{2}} \frac{1}{\sigma_{\mathrm{r}_{i}}^{2}} \frac{1}{\sigma_{\mathrm{p}_{i}}^{2}} \frac{1}{\sigma_{\mathrm{y}_{\mathrm{i}}}^{2}} \frac{1}{\sigma_{\rho_{i}}^{2}} \frac{1}{\sigma_{\theta_{i}}^{2}}\right)
\end{aligned}
$$

and $P_{c, \mathcal{C}}=\left(1 / \sigma_{c}^{2}\right) I$. It is assumed that the constraints for all planes are to be weighted equally. Note that the variance of the constraint $\sigma_{c}^{2}$ must be chosen much smaller than the variances of the measurements for the 'weighted parameter constraint' model to be effective.

\subsection{Least squares solution}

The solution is derived using the traditional approach of least-squares adjustment when dealing with the combined model. In our particular case, the scalar variation or objective function to minimise is the sum of weighted squares of the residuals (of observations and constraints) subject to the constraints of the two functional models. Following standard procedures, the resulting final form of the normal equations used herein is:

$$
\begin{gathered}
{\left[\begin{array}{cc}
A_{1}^{T}\left(B P^{-1} B^{T}\right)^{-1} A_{1} & A_{1}^{T}\left(B P^{-1} B^{T}\right)^{-1} A_{2} \\
A_{2}^{T}\left(B P^{-1} B^{T}\right)^{-1} A_{1} & A_{2}^{T}\left(B P^{-1} B^{T}\right)^{-1} A_{2}+G^{T} P_{c} G
\end{array}\right]\left[\begin{array}{c}
\hat{\delta}_{1} \\
\hat{\delta}_{2}
\end{array}\right]} \\
+\left[\begin{array}{c}
A_{1}^{T}\left(B P^{-1} B^{T}\right)^{-1} w \\
A_{2}^{T}\left(B P^{-1} B^{T}\right)^{-1} w+G^{T} P_{c} w_{c}
\end{array}\right]=\left[\begin{array}{l}
0 \\
0
\end{array}\right]
\end{gathered}
$$

or, in abbreviated notation $N \hat{\delta}+u=0$.

The ALS instruments operate with high data rates that vary between 10 and $150 \mathrm{kHz}$ depending from the type. Considering 8 observations per point, the size of the design matrices can quickly reach a size that is not 


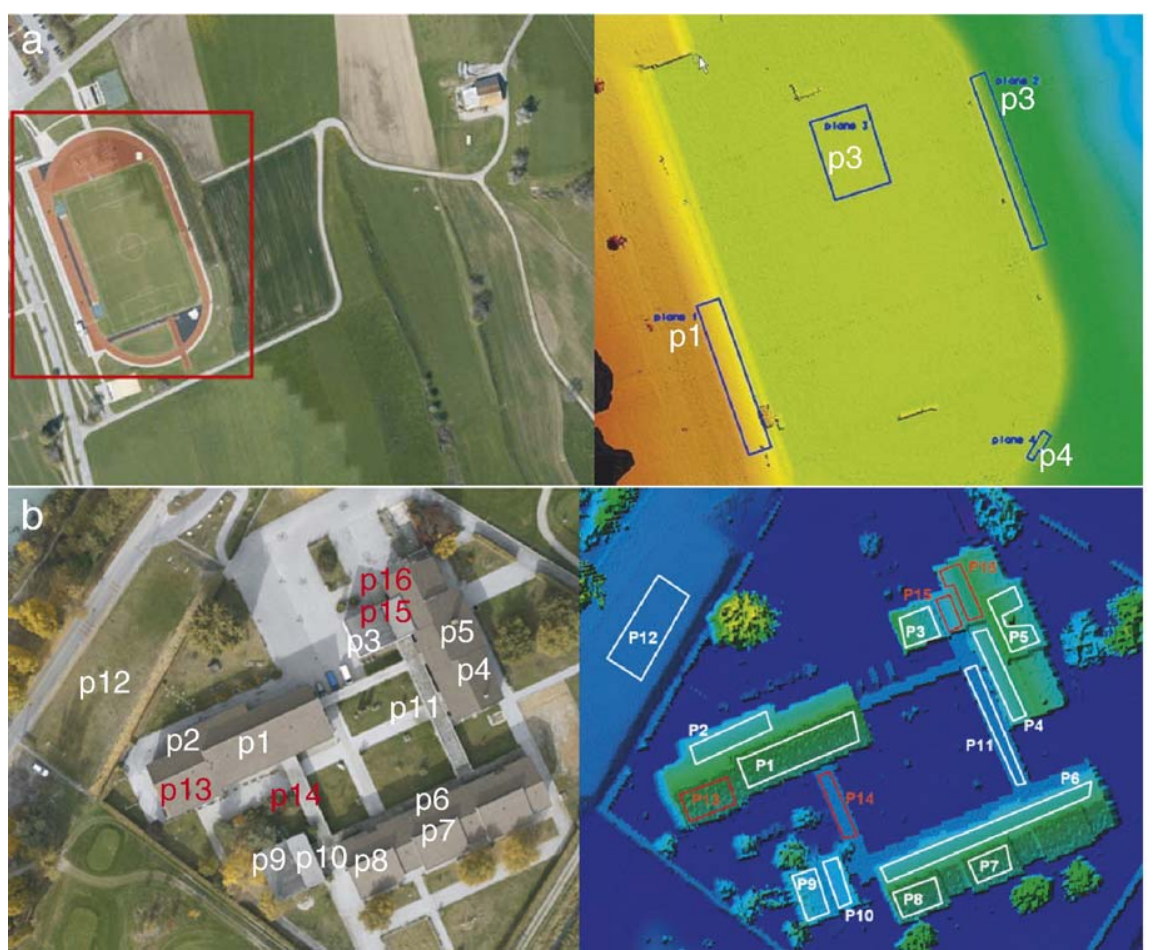

Fig. 2. Images and color-coded reliefs of the two test fields with highlighted planar features: (a) soccer field, (b) urban area (only plane nos. 2-12 were used in the adjustment while the plane nos. 13-16 served for control purposes).

practical for manipulation. Hence, it is worth exploiting the sparse structure of the design matrices and formulating a contribution of individual observation equations in such a way that they can be added directly to the normal equations in a sequential manner. Once the normal equations are formed, the parameters $\hat{\delta}_{1}$ and $\hat{\delta}_{2}$ are computed from Eq. (10) as $\hat{\delta}=-N^{-1} u$. The other estimable quantities like residuals are then computed by back-substitution. The parameter estimates are further improved iteratively. For the iterative solution to start, the approximate calibration parameters (bore-sight angles and the range-finder offset) can be set to zero because these are expected to have relatively small values. However, good approximate plane parameters must be derived. This can be done using the method of orthogonal regression. As shown in Shakarji (1998) the least-squares solution for the normal vector $\vec{n}$ belonging to the plane $j$ reduces to the eigenvalue problem of the covariance matrix, $C$, with all $m$ points $\overrightarrow{r_{i}}$ belonging to the plane $j$ reduced to their centroid:

$$
\begin{aligned}
C \vec{n} & =\left\{\sum_{i=1}^{m}\left(\vec{r}_{i} \vec{r}_{i}^{\mathrm{T}}\right)\right\} \vec{n}=\sum_{i=1}^{m}\left[\begin{array}{ccc}
x_{i}^{2} & x_{i} y_{i} & x_{i} z_{i} \\
x_{i} y_{i} & y_{i}^{2} & y_{i} z_{i} \\
x_{i} z & y_{i} z_{i} & z_{i}^{2}
\end{array}\right]\left[\begin{array}{c}
s_{1_{j}} \\
s_{2_{j}} \\
s_{3_{j}}
\end{array}\right] \\
& =\lambda \vec{n}
\end{aligned}
$$

Since $C$ is a symmetric, positive semi-definite matrix, i.e. all its three eigenvalues, $\lambda$, are real and greater than or equal to zero, the sum of squares of orthogonal distances is minimised when the smallest eigenvalue is selected. Once the normal vector has been computed, the orthogonal distance from the coordinate origin to the plane, $s_{4}$ (negative using this definition), is calculated as $s_{4_{j}}=-\vec{n} T \vec{r}$. The principal advantage of this method is its computational efficiency and thus practicality when using large ALS data sets.

Table 1

\begin{tabular}{|c|c|c|}
\hline Test area & Soccer field & Urban \\
\hline Size & $110 \mathrm{~m} \times 120 \mathrm{~m}$ & $110 \mathrm{~m} \times 70 \mathrm{~m}$ \\
\hline $\begin{array}{l}\text { Height } \\
\text { disparity }\end{array}$ & $9 \mathrm{~m}$ & $7 \mathrm{~m}$ \\
\hline $\begin{array}{l}\text { Flight } \\
\text { pattern }\end{array}$ & $\begin{array}{l}1 \times \text { cloverleaf }(4 \text { lines }) \\
\text { at relative flying } \\
\text { heights between } 180 \mathrm{~m} \\
\text { and } 210 \mathrm{~m}\end{array}$ & $\begin{array}{l}2 \times \text { cloverleaf ( } 8 \text { lines }) \\
\text { at two relative flying } \\
\text { heights: } \sim 150 \mathrm{~m} \\
\text { and } \sim 250 \mathrm{~m}\end{array}$ \\
\hline $\begin{array}{l}\text { Planar } \\
\text { segments }\end{array}$ & 3 (ground) & 11 (roofs and ground) \\
\hline $\begin{array}{l}\text { Plane } \\
\text { orientation } \\
\text { and slope }\end{array}$ & $\begin{array}{l}\text { Limited ( } 1 \text { horizontal, } \\
2 \text { inclined in similar } \\
\text { direction) }\end{array}$ & $\begin{array}{l}\text { Several } \\
\text { slopes (up to } 20^{\circ} \text { ) and } \\
\text { orientations }\end{array}$ \\
\hline $\begin{array}{l}\text { Point } \\
\text { observations }\end{array}$ & $\sim 14,000$ & $\sim 18,000$ \\
\hline
\end{tabular}

Characteristics of used data sets 


\section{Experimental description}

\subsection{System used}

The numerical examples will make use of a compact remote-sensing system built at EPFL (Skaloud et al., 2005) that integrates high accuracy navigation sensors (GPS/INS) with an ALS and high-resolution digital (CCD) camera. The system is operated from the side of a helicopter and is tailored for corridor and large scale/ small area mapping requiring high resolution and accuracy. Thanks to the custom design of the system, all aspects of data flow and processing are controlled from acquisition at the sensor level to final DSM/DTM/ orthophoto generation. Apart from the LiDAR and digital camera, the sensor head incorporates an LN200/ A1 tactical grade IMU with $400 \mathrm{~Hz}$ measurement rate from Northtrop Grumann and a dual-frequency GPS receiver (Javad Legacy GD) with the airborne antenna mounted on a carbon mast that can change orientation with respect to LiDAR/camera plane in fixed intervals from $15^{\circ}$ to $90^{\circ}$ according to mapping requirements. The lever-arm separation between the individual instruments is determined to better than $1 \mathrm{~cm}$ by laboratory calibration and is independent of system installation. The ALS is a short-range 2D scanner (Riegl LMS-Q240) with a scanning angle of $60^{\circ}$ and maximal range of $450 \mathrm{~m}$ at $80 \%$ reflectance. Its rotating-mirror mechanism provides linear, unidirectional and parallel scan lines with a programmable rate. The rate is chosen as a function of desired point density and flight parameters, typically with several points per $\mathrm{m}^{2}$. Contrary to most of today's airborne scanners, this instrument adapts a relatively short laser wavelength of $900 \mathrm{~nm}$ and a range-finder that is specified to maintain $5 \mathrm{~cm}$ resolution and $3 \mathrm{~cm}$-level accuracy under optimal conditions.

\subsection{Calibration fields}

In the following analyses, we make use of the two test fields depicted in Fig. 2. The first is essentially the flat terrain of a soccer field with gently-inclined slopes in its vicinity. The second calibration field was chosen over a group of buildings with rooftops of different slope and aspect. More details about the properties of the captured data sets are listed in Table 1. As will become apparent, the differences between the two terrain types will serve to demonstrate the effects of weak/strong geometry on the quality of the calibrated parameters when applying the proposed method.

The GPS conditions were almost optimal for both data sets, with favorable satellite geometry and close separation from the reference receiver. The flight lines were short (few minutes) and high-dynamic turns were executed between them. The rapid change in direction and the increased horizontal acceleration makes the individual systematic errors within the inertial system well observable by GPS position and velocity updates while operating flight lines of short duration limits their accumulation. These facts, together with the low-flying height, contributed to the good determination of the GPS/INS trajectory ( $\sigma$ position per coordinate $<5 \mathrm{~cm}, 1 \sigma$ orientation $<0.01^{\circ}$ ).

\section{Solution analyses}

\subsection{Parameter estimates}

After generating the laser point clouds for both data sets, the planar features had to be selected. The assumption of their planarity (i.e. no departures greater than the noise level of surface measurements) is central to this method and especially those that use natural terrain (e.g. Filin, 2003). In case of the urban data set, such a classification can be quickly performed by manual 'fence drawing'. Finding the planar features in the 'natural' terrain requires the use of special classification routines or visual guidance by means of orthophoto or approximate elevation models. Our experience in this regard has been that finding suitable natural terrain even on such 'planar' surfaces like soccer field is problematic. Only small patches could be considered to be sufficiently flat.

Table 2

Influence of plane geometry and range-finder correction on bore-sight estimation

\begin{tabular}{|c|c|c|c|c|c|c|c|c|}
\hline & \multicolumn{4}{|c|}{ With $\Delta \rho$} & \multicolumn{4}{|c|}{ Without $\Delta \rho$} \\
\hline & \multicolumn{2}{|c|}{ Soccer field } & \multicolumn{2}{|l|}{ Urban } & \multicolumn{2}{|c|}{ Soccer field } & \multicolumn{2}{|l|}{ Urban } \\
\hline & Param. & $\sigma$ & Param. & $\sigma$ & Param. & $\sigma$ & Param. & $\sigma$ \\
\hline$\alpha\left(^{\circ}\right)$ & 0.138 & 0.0007 & 0.137 & 0.0008 & 0.136 & 0.0006 & 0.139 & 0.0007 \\
\hline$\beta\left(^{\circ}\right)$ & -0.069 & 0.0009 & -0.059 & 0.001 & -0.066 & 0.0008 & -0.060 & 0.0009 \\
\hline$\gamma\left({ }^{\circ}\right)$ & -0.036 & 0.011 & -0.076 & 0.010 & -0.106 & 0.009 & -0.057 & 0.009 \\
\hline$\Delta \rho(\mathrm{m})$ & -0.158 & 0.016 & 0.109 & 0.022 & - & - & - & - \\
\hline
\end{tabular}


Table 3

Plane fit results before and after calibration

\begin{tabular}{lcll}
\hline Plane ID & Number of points & $\sigma$ (before; $\mathrm{m})$ & $\sigma$ (after; $\mathrm{m})$ \\
\hline 2 & 1728 & 0.042 & 0.003 \\
3 & 714 & 0.038 & 0.003 \\
4 & 1546 & 0.045 & 0.002 \\
5 & 1410 & 0.045 & 0.003 \\
6 & 3366 & 0.047 & 0.002 \\
7 & 1585 & 0.047 & 0.003 \\
8 & 1366 & 0.049 & 0.003 \\
9 & 990 & 0.040 & 0.002 \\
10 & 678 & 0.041 & 0.003 \\
11 & 1345 & 0.045 & 0.001 \\
12 & 3523 & 0.035 & 0.003 \\
\hline
\end{tabular}

Although not investigated here, it is very likely that the parameters most influenced by a warped surface would be $s_{4}$ and $\Delta \rho$. Together with the target coordinates and GPS/INS trajectory, the point-cloud classification is the only pre-requisite to the model input. The initial parameter values for the planes are then found directly from the data, and the rest of the adjustment can follow the described automated procedure.
Table 2 depicts the system-parameter estimates for both calibration fields and range-finder offset included as an option. As can be seen from the table, the inclusion of the range-finder parameter has a serious influence on the bore-sight estimate and the $\Delta \rho$ magnitude appears significant when considering the sigma-to-value ratio. This can be partially explained by the existing moderate correlation $(\sim 0.4)$ between $\Delta \rho$ and all bore-sight angles. Furthermore, the estimated magnitude of $\Delta \rho$ varies between data sets in the decimeter range which is outside the LiDAR accuracy specification $(1 \sigma=2.5 \mathrm{~cm})$. The high estimates of $\Delta \rho$ were later confirmed to be unrealistic by independent static testing using electronic distance measurements as a reference. There, no statistically significant discrepancies from the given instrument specifications were found. Therefore, its presence in the adjustment needs to be traced to a different cause, as will be done later on. The second important observation is the difference in $\gamma$ (heading) estimation between both data sets. This is most likely caused by the weak geometry of the soccer-field terrain, with one horizontal and two gently inclined slopes of the
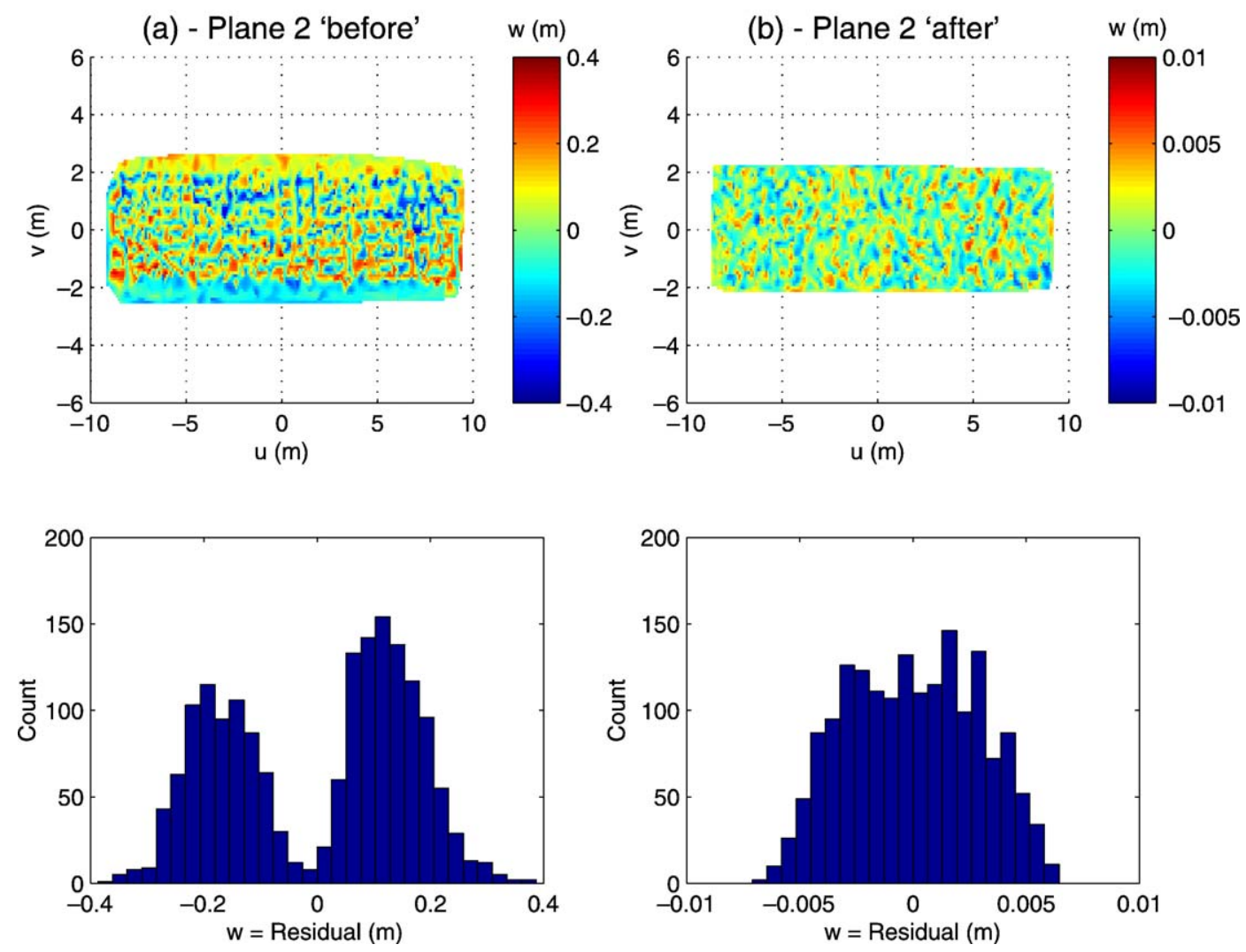

Fig. 3. The surface plots of the plane-fit residuals with corresponding histograms: (a) before and (b) after adjustment. 
Table 4

Independent plane fit results before and after calibration

\begin{tabular}{llll}
\hline Plane ID & Number of points & $\sigma$ (before; $\mathrm{m})$ & $\sigma$ (after; m) \\
\hline 13 & 1361 & 0.107 & 0.060 \\
14 & 735 & 0.053 & 0.040 \\
15 & 413 & 0.078 & 0.043 \\
16 & 894 & 0.082 & 0.039 \\
\hline
\end{tabular}

same aspect. The bore-sight angles $\alpha$ and $\beta$ are less sensitive in this regard and are also more precisely estimated. The subsequent analysis will therefore focus on the urban data set that has a good geometry thanks to significant variations in plane inclination and aspect.

\subsection{Planar surface analysis}

This section quantifies the impact of applying the estimated bore-sight parameters with respect to the planar surfaces. Table 3 shows standard deviations of plane fits computed from the individual point clouds by orthogonal regression before and after application of the parameters from the proposed calibration method. Also,

(a) - Plane 16 'before'
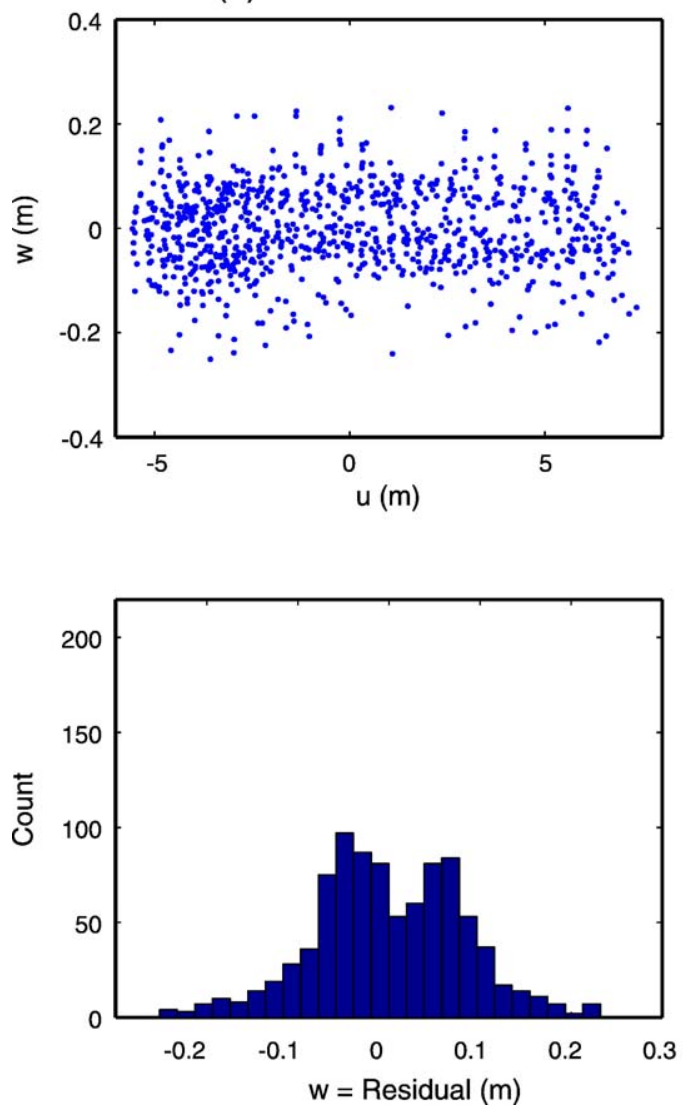

the numbers of points per plane are indicated for reference. Clearly, these results demonstrate that large improvement-an order of magnitude-was consistently achieved. The following plots offer more insight into the distributions of the out-of-plane residuals before and after the adjustment.

Fig. 3a (top) shows a representative surface plot of plane-fit residuals before application of the calibration parameters for plane No. 2. The point cloud is plotted in plane-centric coordinate system in which the origin is at the roof centroid and the $u$-, $v$ - and $w$-axes correspond to the principal axes from the planar point-cloud covariance-matrix eigenvalue decomposition. Correlation in the residuals is evident in the mottled texture of the 'out-of-surface' $w$-axis and the linear trends in these patterns and high magnitude of errors (up to $\pm 0.4 \mathrm{~m}$ ) indicate systematic effects. Fig. 3a (bottom) shows the histogram of the residuals for plane No. 2 without calibration correction. The point clouds show two distinct surfaces caused by un-modeled bore-sight errors that correlate to flight direction and explain the bimodal histograms.

(b) - Plane 16 'after'
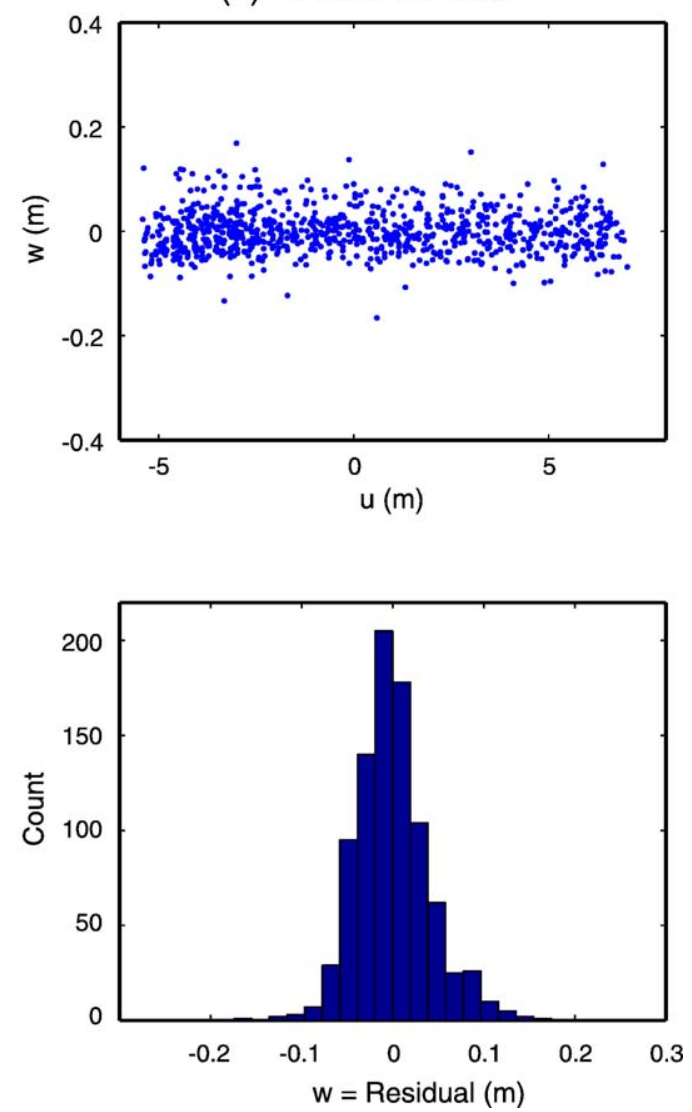

Fig. 4. The point cloud plots of the plane-fit residuals with corresponding histograms: (a) before and (b) after adjustment. 
After-calibration results for plane No. 2 are shown in Fig. 3b. The first observation of the surface-plot residuals is the lower magnitude of the residual range ( $\pm 6 \mathrm{~mm}$ ). Though the texture of this surface suggests the presence of spatial correlation, presumably caused by some temporal correlation process, it occurs at a relatively high spatial frequency so it is not due to unmodeled bore-sight angles. This is further confirmed by analyzing the histogram of Fig. $3 \mathrm{~b}$ (bottom). Here there is a single surface visible in the point cloud and the histogram is uni-modal. Though the histogram is not exactly Gaussian-shaped, systematic errors due to boresight have been removed and those that remain are well below the system noise level.

Four additional planes from the same site were also extracted to independently evaluate the success of the calibration method. These planes are generally smaller and were not used in the adjustment. Presented in Table 4 are the 'before' and 'after' residual standard deviations from these planes. The after-calibration values are more realistic and correspond to the theoretical system noiselevel of $\sim 5 \mathrm{~cm}$ due to the fact that the measurements related to these planes were not adjusted. Therefore, the improvement is not as large as in Table 4 but is still significant. The notable exception is plane 14 , for which the standard deviation only improves by $1 \mathrm{~cm}$. This is not unexpected since this plane is horizontal and, as demonstrated in Fig. 1, bore-sight errors are thus more difficult to detect. The improvement gained is best repre-

(a)

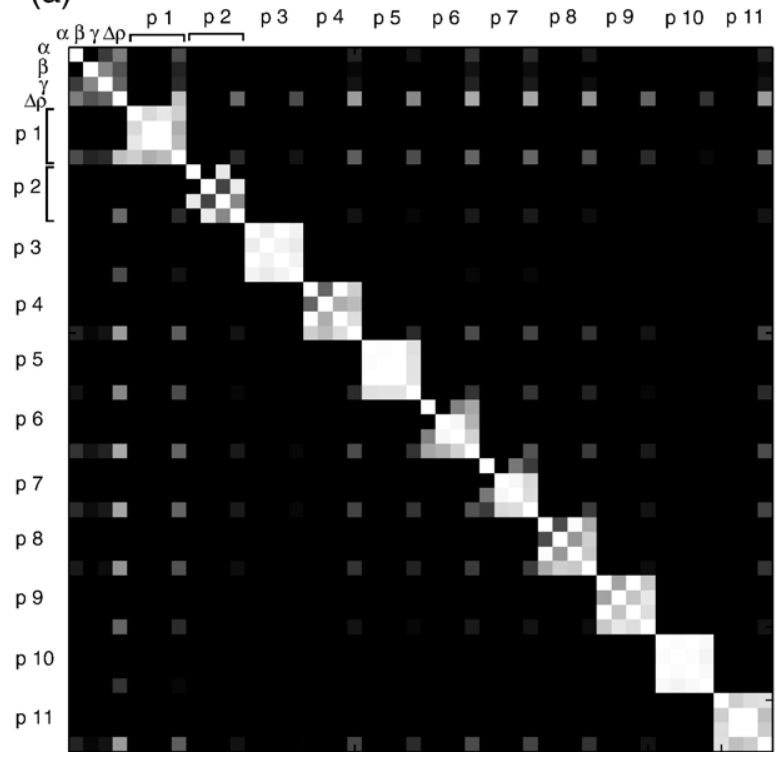

sented by the 'before' and 'after' point clouds and histograms of plane fit residuals given in Fig. $4 \mathrm{a}$ and $\mathrm{b}$, respectively. Similarly to plane No. 2, Fig. 4a (top) shows the un-modeled bore-sight errors as two disparate surfaces, which are more evident in the bimodal structure of the histogram (bottom). Fig. $4 \mathrm{~b}$ shows the effect has been removed in both the point cloud and more Gaussian-like histogram.

\subsection{Parameter correlation}

The correlation between the estimated parameters can be considered as a good indicator of the adjustment quality. Fig. 5 displays the full correlation matrix for the urban data set when including (a) $\Delta \rho$ in the estimated values or not (b). As can be seen from Fig. 5a, $\Delta \rho$ (4th parameter) is not only correlated with all bore-sight angles (first 3 parameters) but also with all $s_{4}$ parameters (the orthogonal distances between the planes and the origin). Such correlations disappear completely once $\Delta \rho$ is eliminated from the adjustment (Fig. 5b). In this case, the estimated bore-sight angles are no longer correlated with the plane parameters as the covariance matrix has a dominant block-diagonal structure. They also de-correlate with respect to each other, although small correlation between the pitch and the heading angles persists. The remaining correlations among the parameters of the individual planes are not important as there is a conditional relation between them. Hence, these results can

(b)

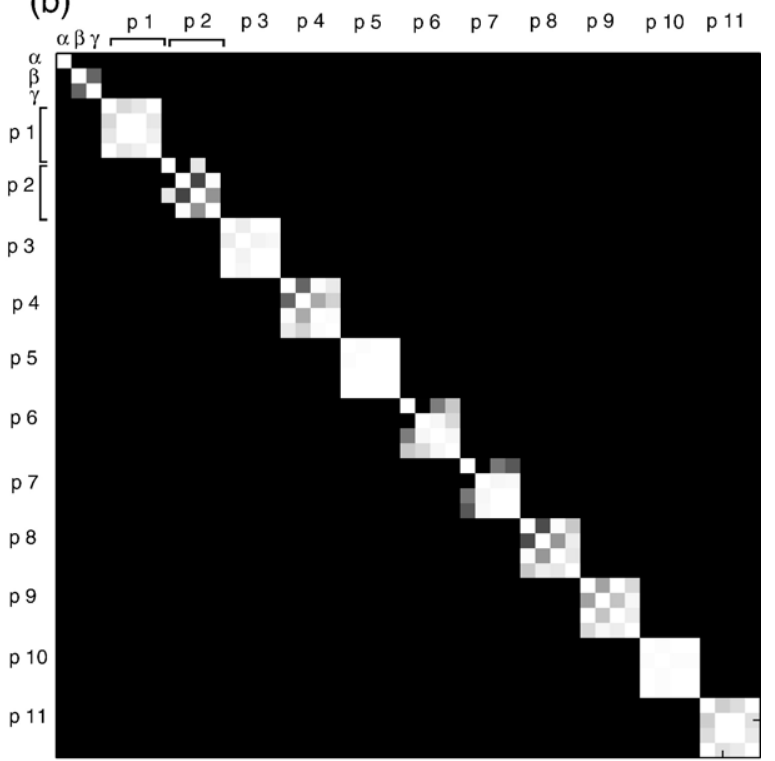

Fig. 5. Correlation between the estimated parameters (3 bore-sight $+4 \cdot q$ planes, $n=11)$ with $\Delta \rho$ included as the optional 4 th parameter (a) and excluded (b). The gray scale varies from black (zero correlation) to white (correlation of \pm 1 ). 

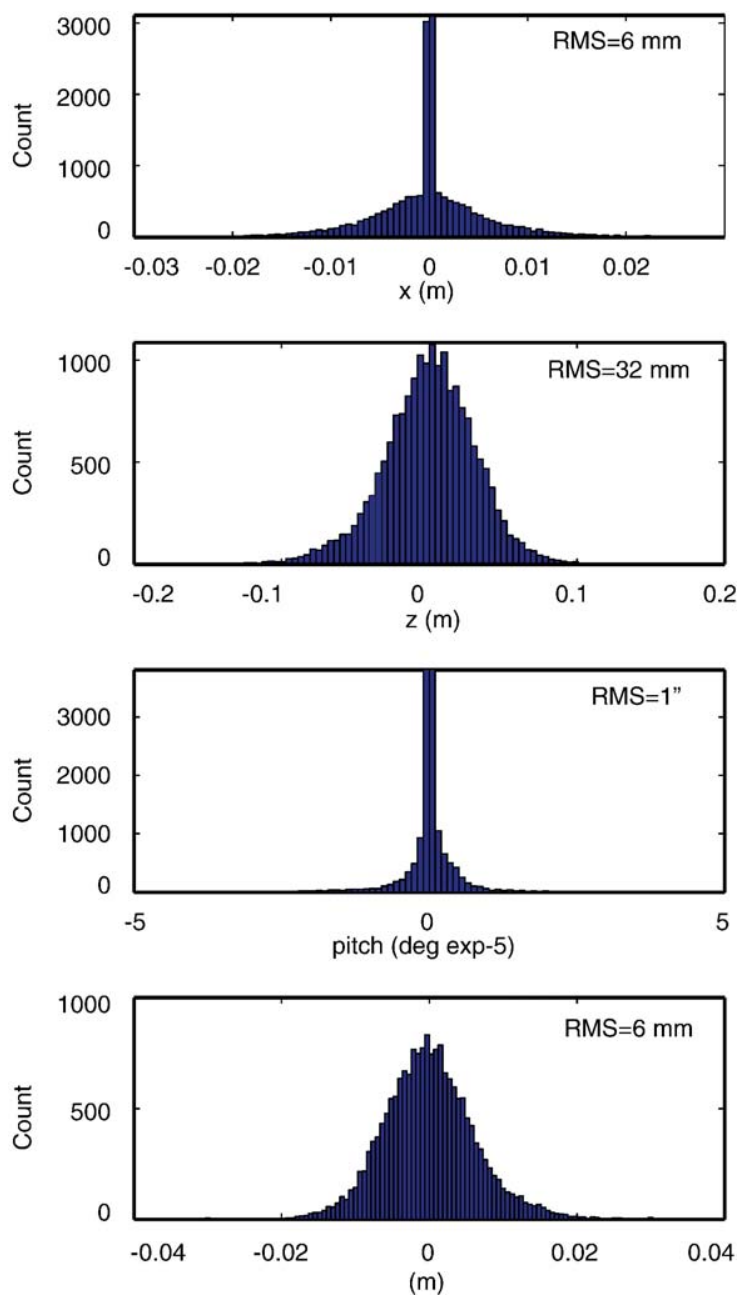

Fig. 6. Histogram of residuals for the urban data (without $\Delta \rho$ estimate), for observations in $x$ and $z$ positions, orientation in pitch and range. Note that for the other observables, the histogram of $y$ is similar to $x$, heading is similar to pitch while roll and encoder-angle histograms show less zeros.

be considered as very satisfactory in terms of parameter de-correlation.

Let us briefly return to the situation where the boresight angles and the range-finder error shall be estimated together. One might argue that a better de-correlation could be achieved by determining the plane parameters by some independent means (Filin, 2003), which would correspond to sacrificing the practical benefits of this approach. However, the authors are less convinced that such approach can yield satisfactory results for highprecision systems when $\Delta \rho$ needs to be recovered with the $\mathrm{cm}$-level accuracy. The limiting factor is probably the positioning noise level of the GPS/INS trajectory where errors of $\sim 0.1 \mathrm{~m}$ magnitude can exist due to differential troposphere error and/or the satellite constellation. In other words, regardless of the contribution of the absolute plane parameter values, the accuracy of the GPSderived height at the flight level will most likely remain the limiting factor when attempting the in-flight recovery of $\Delta \rho$ at the sub-decimeter level. Other contributing factors could be range biases caused by different intensity levels due to different surface reflectivity among the various planes, incidence angle, lower intensity returns at the higher flying height (i.e. power loss due to dependence on the reciprocal of range squared for an extended diffuse target) or a combination of all three. The task of finding a suitable correction function to account for these factors would be quite involved.

\subsection{Model analysis}

The quality of the modeling can be judged from the distribution of observation residuals after the adjustment.

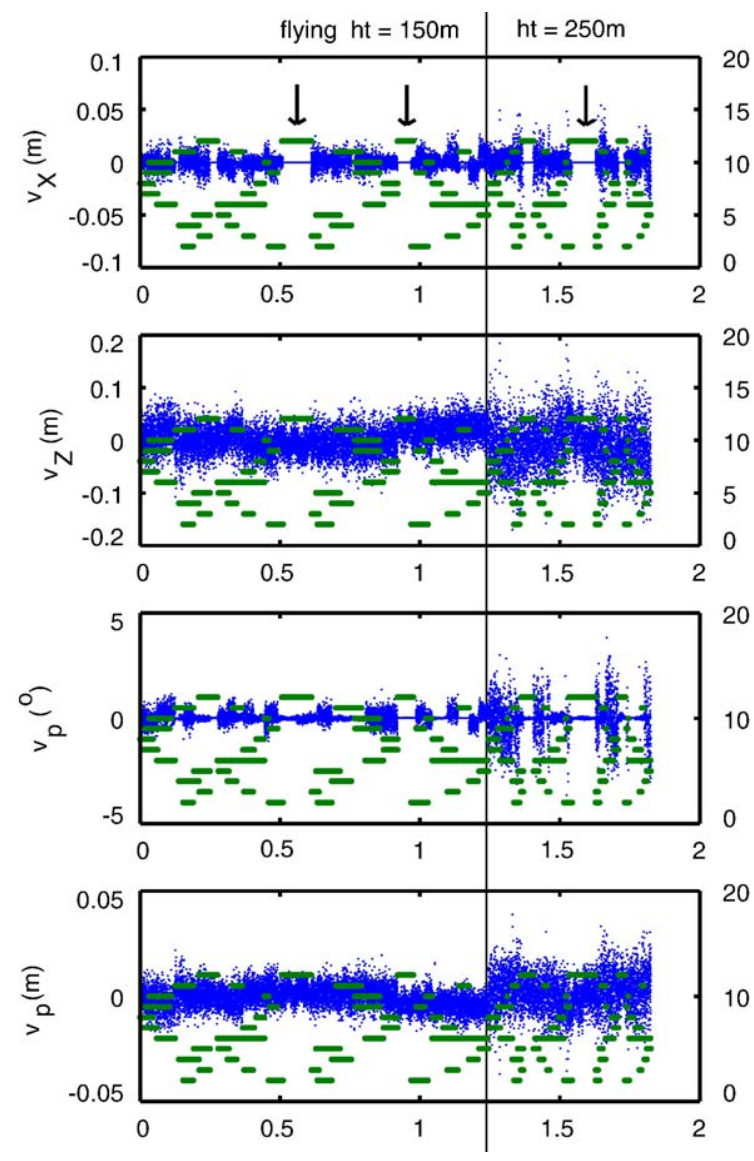

Fig. 7. Magnitude of residuals (left vertical axes) by point index and the indication of the planes that the points belong to (right vertical axes). Due to similarities, the residuals are plotted only in $x$ and $z$ positions, pitch and range. The vertical line indicates the change in the flying height and the higher altitude exhibits an expected increase in noise level. 
Table 5

Adjustment's radius of convergence

\begin{tabular}{|c|c|c|c|c|}
\hline \multicolumn{3}{|c|}{ Approximate values } & \multirow{2}{*}{$\begin{array}{l}\text { No. of } \\
\text { iteration } \\
\text { required }\end{array}$} & \multirow{2}{*}{$\begin{array}{l}\text { Converged } \\
\text { to same } \\
\text { values? }\end{array}$} \\
\hline$\alpha\left(^{\circ}\right)$ & $\beta\left(^{\circ}\right)$ & $\gamma\left({ }^{\circ}\right)$ & & \\
\hline 0 & 0 & 0 & 4 & - \\
\hline 5 & 0 & 0 & 5 & Yes \\
\hline 0 & 5 & 0 & 5 & Yes \\
\hline 0 & 0 & 5 & 5 & Yes \\
\hline 5 & 5 & 5 & 5 & Yes \\
\hline 10 & 10 & 10 & 5 & Yes \\
\hline 20 & 20 & 20 & 6 & Yes \\
\hline 30 & 30 & 30 & 6 & Yes \\
\hline
\end{tabular}

Fig. 6 shows the histogram of the residuals for four series of observables together with their respective RMS. Several important phenomena can be noticed. First of all, the RMS values are fairly small $(\mathrm{mm}$ or $\mathrm{cm}$ level in position and range; arc-second level for the angular quantities) and centered around zero (i.e. they are unbiased). It can therefore be concluded that no important parameters were omitted from the model, and that the overall estimation is in good condition. Secondly, there is significant number of residuals that remain close or equal to zero in certain observations. To explain these occurrences, the residuals are plotted with respect to their magnitude and plane index in Fig. 7. As can be seen from this figure, the zero residuals correlate with the large horizontal planes 11 and 12 (indicated by the arrows). This confirms the previous findings that horizontal planes contribute very little to the estimates of bore-sight angles in pitch and heading direction (or shifts in horizontal position, would they be included). Intuitively, this could be explained as a result of the laser 2D measurement principle where the scan lines are roughly oriented across track and the third dimension is introduced by the motion of the carrier. Note also that the magnitude of residuals increases with the higher flying height as shown in Fig. 7 (indicated by the vertical line).

It is also interesting to compare the absolute values of the bore-sight angles to those estimated by cross-section method. Considering the de-correlated bore-sight estimate from urban data set as a reference (bold numbers in Table 2), a good correspondence is found only in the roll while the value of the pitch differs by $100 \%$ of its magnitude. As previously stated, the cross-section method cannot estimate the bore-sight in heading direction.

\subsection{Stability of convergence}

The sensitivity of the adjustment concerning the approximate calibration values is another important fac- tor for judging method's robustness and applicability. We have assumed the initial bore-sight angles and rangefinder offset to be zero and the approximate plane parameters were derived using all points. In these cases the adjustment converged in 4 iterations with the aposteriori $\hat{\sigma}_{0}^{2}=0.3$. However, what was valid for a particular system where bore-sight angles are 'physically' small cannot be assumed in general. Therefore, a test was designed to determine the adjustment radius of convergence or its 'pull-in range'. For this purpose, the urban calibration field was used and the convergence criterion for all parameters was set to $10^{-5}$. The range-finder offset was not estimated in this case. Table 5 shows the magnitude of different approximate values used for each calibration angle up to the extreme value of $30^{\circ}$. As it can be seen from the last two columns of this table, the estimated parameters always converged to the same values with the small penalty of looping through one or two additional iterations. Hence, the method can be considered as very robust in terms of convergence and largely insensitive to the initial parameter values.

\section{Summary}

We have presented a new method of bore-sight selfcalibration in ALS. Our approach stemmed from modeling the systematic influences on the sensor level and within the direct-georeferencing equations for each target point and conditioning groups of points to lie on a common surface. Using planes as the parametric surfaces is not only easy to implement but also practical as these are abundant in urban areas and man-made structures. Though a similar approach has been recently published elsewhere, results with real data have not been published. Contrary to other previous work in this area, the absolute plane parameters need not be supplied by other means and are recovered together with the system parameters. Hence, many and even planes not directly accessible can be used within the calibration field. However, to achieve sufficient de-correlation between the plane and system parameters it is important that the planes vary in slope and orientation. Furthermore, the estimation was formulated in a way that allows convenient handling of voluminous ALS data. The proposed model can be extended in a straightforward manner to include other systematic errors either of the LiDAR or of the navigation data; yet, such augmentation may result in strong correlation among some parameters as was demonstrated for the range-finder correction. In the presented cases, the recovery of $\Delta \rho$ was limited by the residual biases in GPS-height at the 1-dm level. The effect of absolute height accuracy on the range error estimate can be, at 
least theoretically, reduced if vertically orientated planes are also observed. However, this is possible only in the particular case of the presented system that is pointed manually and does not apply to ALS in general. On the other hand, the control of the range-finder performance can be performed by static testing, as was done in our case. Hence, the conditions for the calibration flight should be chosen as favorable as possible for the GPS/ INS integration and other systematic influences in LiDAR observations shall be estimated by independent means whenever feasible. The numerical examples showed that RMS residuals as low as $3 \mathrm{~cm}$ in height of GPS/INS, 2 arc seconds in angles and $1 \mathrm{~cm}$ in laser-range can be achieved with a precise close-range ALS system after the correct recovery of systematic sources. Though the testing has been conducted using a continuouslyrotating mirror system, the proposed method's mathematics have been formulated independent of scanning mechanism. Further testing is required to confirm its benefit to oscillating-mirror systems, for example.

\section{Acknowledgements}

This project was partly funded by the Swiss Commission for Innovation (CTI/KTI Project 7782.1 EPRP) in collaboration with Swissphoto AG attributed to the first author as well as internal funds from EPFL and Curtin University of Technology. The flying expertise was provided by Dr. Julien Vallet, UW $+\mathrm{R}$ and Air Glacier, Switzerland. These contributions are greatly appreciated. The authors also thank Dr. Klaus Legat and Philipp Schaer of EPFL-TOPO for their help in data capture and classification and manuscript review.

\section{References}

Burman, H., 2000. Calibration and Orientation of Airborne Image and Laser Scanner Data Using GPS and INS, PhD Thesis, Photogrammetry Reports No. 69, Royal Institute of Technology, Stockholm, 107 p.

Cramer, M., Stallmann, D., 2002. System calibration for direct georeferencing. International Archives of Photogrammetry, Remote Sensing and Spatial Information Sciences 34 (Part 3A), 79-84.
Filin, S., 2003. Recovery of systematic biases in laser altimetry data using natural surfaces. ISPRS Journal of Photogrammetric Engineering and Remote Sensing 69 (11), 1235-1242.

Filin, S., Vosselman, G., 2004. Adjustment of airborne laser altimetry strips. International Archives of Photogrammetry, Remote Sensing and Spatial Information Sciences 34 (Part B3), 285-289.

Förstner, W., Wrobel, B., 2004. Mathematical concepts in photogrammetry, In: McGlone, J.C., Mikhail, E.M., Bethel, J., Mullen, R. (Eds.), Manual of Photogrammetry, fifth ed. American Society of Photogrammetry and Remote Sensing, Bethesda, MA, pp. 15-180.

Friess, P., 2006. Toward a rigorous methodology for airborne laser mapping. Proceedings International Calibration and Orientation Workshop EuroCOW 2006, Castelldefels, Spain, 25-27 January, 7 p. (on CDROM).

Katzenbeisser, R., 2003. About the calibration of lidar sensors. International Archives of Photogrammetry, Remote Sensing and Spatial Information Sciences 34 (Part 3/W13), 59-64.

Kruck, E., 2001. Combined IMU and sensor calibration with BINGOF. Proceedings OEEPE Workshop on Integrated Sensor Orientation, Hannover, Germany, 1-3 March, 11 p. (on CDROM).

Lichti, D., 2004. A resolution measure for terrestrial laser scanners. International Archives of Photogrammetry, Remote Sensing and Spatial Information Sciences 34 (Part B5), 552-558.

Morin, K., El-Sheimy, N., 2002. Post-mission adjustment of airborne laser scanning data, Proceedings XXII FIG International Congress, Washington DC, USA, 19-26 April, 12 p. (on CDROM).

Optech, 2004. ALTM 30/70/100 User Manual, Toronto, Canada.

Schenk, T., 2001. Modeling and Analyzing Systematic Errors in Airborne Laser Scanners. Technical Notes in Photogrammetry, vol. 19. The Ohio State University, Columbus, USA. 46 p.

Shakarji, C.M., 1998. Least-squares fitting algorithms of the NIST algorithm testing system. Journal of Research of the National Institute of Standards and Technology 103 (6), 633-641.

Skaloud, J., Schaer, P., 2003. Towards a more rigorous boresight calibration, Proceedings ISPRS International Workshop on Theory Technology and Realities of Inertial/GPS/Sensor Orientation, Castelldefels, Spain, 22-23 September, 9 p. (on CDROM).

Skaloud, J., Vallet, J., Keller, K., Veyssiere, G., Koelbl, O., 2005. HELIMAP: Rapid large scale mapping using handheld LiDAR/CCD/ GPS/INS sensors on helicopters, Proceedings ION GNSS 2005, Long Beach, CA, USA, 13-16 September, pp. 2461-2467 (on CDROM).

Soininen, A., Burman, H., 2005. TerraMatch for MicroStation. Terrasolid Ltd, Finland.

Vosselman, G., Maas, H.-G., 2001. Adjustment and filtering of raw laser altimetry data. Proceedings OEEPE Workshop on Airborne Laser scanning and Interferometric SAR for Detailed Digital Elevation Models. OEEPE Publication, vol. 40, pp. 62-72. Stockholm, Sweden. 